International Journal of Pure and Applied Mathematics

Volume 111 No. 2 2016, 273-280

ISSN: 1311-8080 (printed version); ISSN: 1314-3395 (on-line version)

url: http://www.ijpam.eu

doi: 10.12732/ijpam.v111i2.11

\title{
RICCI AND PROJECTIVE CURVATURE TENSORS ON A TYPE OF PARA-KENMOTSU MANIFOLD
}

\author{
S. Sunitha Devi ${ }^{1}$, K.L. Sai Prasad ${ }^{2} \S$, G.V.S.R. Deekshitulu ${ }^{3}$ \\ ${ }^{1}$ Department of Mathematics \\ Vignan Institute of Information Technology \\ Visakhapatnam, 530 049, INDIA \\ ${ }^{2}$ Department of Mathematics \\ Gayatri Vidya Parishad College of Engineering for Women \\ Madhurawada, Visakhapatnam, 530 048, INDIA \\ ${ }^{3}$ Department of Mathematics \\ Jawaharlal Nehru Technological University \\ Kakinada, 533 003, INDIA
}

\begin{abstract}
The object of the present paper is to study the curvature properties of Ricciparallel para-Kenmotsu (briefly, $P$-Kenmotsu) manifold with the conditions $R(X, \xi) \cdot P=$ $P(X, \xi) \cdot R$ and $R(X, \xi) \cdot P=L[(X \wedge \xi) \cdot P],(L \neq-1)$, where $R(X, Y)$ is the Riemannian curvature tensor, $P(X, Y)$ is the Weyl-projective curvature tensor, $\mathrm{L}$ is some function on $M_{\mathrm{n}}$ and $\mathrm{X} \in \mathrm{T}\left(M_{\mathrm{n}}\right)$. It is shown that the Ricci-parallel $P$-Kenmotsu manifold with these conditions is of constant curvature -1 and consequently it is locally isomorphic to the hyperbolic space. Further, the para-Kenmotsu manifold with the condition $R(\xi, X) \cdot R=0$ is considered and it is shown that such a manifold is of constant curvature. Finally, it is shown that the para-Kenmotsu manifold with the condition $R(\xi, X) \cdot S=0$ is an Einstein manifold, where $S(X, Y)$ is the Ricci curvature tensor.
\end{abstract}

AMS Subject Classification: 53C15, 53C25, 53C50

Key Words: curvature tensor, Ricci tensor, Ricci-parallel para-Kenmotsu manifold, Einstein manifold

Received: $\quad$ September 21, 2016

Revised: $\quad$ October 21, 2016

Published: $\quad$ December 11, 2016

$\S$ Correspondence author (c) 2016 Academic Publications, Ltd. url: www.acadpubl.eu 


\section{Introduction}

The notion of an almost para contact Riemannian manifold was introduced by Sato [1], in 1976. After that, Adati and Matsumoto [2] defined and studied $P$-Sasakian and SP-Sasakian manifolds which are regarded as a special kind of an almost contact Riemannian manifolds. Before Sato, in 1972, Kenmotsu [3] defined a class of almost contact Riemannian manifold. In 1995, Sinha and Sai Prasad [4] defined a class of almost para contact metric manifold namely para-Kenmotsu (briefly, $P$-Kenmotsu) and special para-Kenmotsu (briefly, $S P$-Kenmotsu) manifolds. Later, Satyanarayana and Sai Prasad [5, 6] have studied the curvature properties of semi-symmetric and Ricci-symmetric para-Kenmotsu manifolds.

In this paper, we consider Ricci-parallel para-Kenmotsu manifolds with the conditions $R(X, \xi) \cdot P=P(X, \xi) \cdot R$ and $R(X, \xi) \cdot P=L[(X \wedge \xi) \cdot P], L \neq-1$ to obtain some of their curvature properties. Further, the para-Kenmotsu manifolds with the conditions $R(\xi, X) \cdot R=0$ and $R(\xi, X) \cdot S=0$ are also considered and their curvature properties are studied.

Let $M_{n}$ be an $n$-dimensional differentiable manifold equipped with structure tensors $(\Phi, \xi, \eta)$, where $\Phi$ is a tensor of type $(1,1), \xi$ is a vector field, $\eta$ is a 1 -form such that

$$
\begin{gathered}
\eta(\xi)=1 \\
\Phi^{2}(X)=X-\eta(X) \xi ; \bar{X}=\Phi X .
\end{gathered}
$$

Then $M_{n}$ is called an almost para contact manifold.

Let $g$ be the Riemannian metric satisfying such that, for all vector fields $X$ and $Y$ on $M_{n}$,

$$
\begin{gathered}
g(X, \xi)=\eta(X) \\
\Phi \xi=0, \eta(\Phi X)=0, \operatorname{rank} \Phi=n-1 \\
g(\Phi X, \Phi Y)=g(X, Y)-\eta(X) \eta(Y) .
\end{gathered}
$$

Then the manifold $M_{n}[1]$ is said to admit an almost para contact Riemannian structure $(\Phi, \xi, \eta, g)$.

A manifold $M_{n}$ of dimension $n$ with Riemannian metric $g$ admitting a tensor field $\Phi$ of type (1, 1), a vector field $\xi$ and a 1-form $\eta$ satisfying (1.1), (1.3) along with

$$
\begin{gathered}
\left(\nabla_{X} \eta\right) Y-\left(\nabla_{Y} \eta\right) X=0 \\
\left(\nabla_{X} \nabla_{Y} \eta\right) Z=[-g(X, Z)+\eta(X) \eta(Z)] \eta(Y)+[-g(X, Y)+\eta(X) \eta(Y)] \eta(Z) \\
\nabla_{X} \xi=\Phi^{2} X=X-\eta(X) \xi
\end{gathered}
$$




$$
\left(\nabla_{X} \Phi\right) Y=g(\Phi X, Y) \xi-\eta(Y) \Phi X
$$

is called a para-Kenmotsu manifold or in brief $P$-Kenmotsu manifold [4], where $\nabla$ is the covariant differentiation with respect to the metric $g$.

Let $\left(M_{n}, g\right)$ be an $n$-dimensional Riemannian manifold admitting a tensor field $\Phi$ of type $(1,1)$, a vector field $\xi$ and a 1-form $\eta$ satisfying

$$
\left(\nabla_{X} \eta\right) Y=g(X, Y)-\eta(X) \eta(Y)
$$

$$
g(X, \xi)=\eta(X) \text { and }\left(\nabla_{X} \eta\right) Y=\varphi(\bar{X}, Y) \text {, where } \varphi \text { is an associate of } \Phi \text {. }
$$

Then $M_{n}$ is called special para-Kenmotsu manifold or in brief $S P$-Kenmotsu manifold [4].

It is known that, in a P-Kenmotsu manifold the following relations hold $\operatorname{good}[4]$ :

$$
\begin{gathered}
S(X, \xi)=-(n-1) \eta(X), \text { where } g(Q X, Y)=S(X, Y) \\
g[R(X, Y) Z, \xi]=\eta[R(X, Y, Z)]=g(X, Z) \eta(Y)-g(Y, Z) \eta(X) \\
R(X, \xi)=-1 \\
R(\xi, X) \xi=X-\eta(X) \xi \\
R(X, \xi, X)=\xi \\
R(X, Y, \xi)=\eta(X) Y-\eta(Y) X ; \text { when } X \text { is orthogonal to } \xi \\
R(\xi, X) Y=\eta(Y) X-g(X, Y) \xi ;
\end{gathered}
$$

where $Q$ is the Ricci operator.

An almost para-contact Riemannian manifold $M_{n}$ is said to be an $\eta$-Einstein manifold [7] if the Ricci curvature tensor $S$ is of the form

$$
S=a I_{d}+b \eta \otimes \xi
$$

where $a$ and $b$ are smooth functions on $M_{n}$. In particular, if $b=0$ then it is said to be an Einstein manifold [7].

Moreover, it is also known that if a P-Kenmotsu manifold is projectively flat then it is an Einstein manifold and the scalar curvature has a negative constant value $-n(n-1)$. Especially, if a P-Kenmotsu manifold is of constant curvature, the scalar curvature has a negative constant value $-n(n-1)$ [4] and in this case

$$
\begin{gathered}
S(Y, Z)=-(n-1) g(Y, Z) \\
S(\Phi Y, \Phi Z)=S(Y, Z)+(n-1) \eta(Y) \eta(Z) \text { and } \\
R(X, Y, Z, W)=\frac{1}{(n-1)}[S(Y, Z) g(X, W)-S(X, Z) g(Y, W)] .
\end{gathered}
$$




\section{P-Kenmotsu Manifold with the condition $R(\xi, X) \cdot R=0$}

The endomorphisms $(X \wedge Y),\left(X \wedge_{s} Y\right)$, and the homeomorphism $R(\xi, X) \cdot R$ are respectively defined as:

$$
\begin{gathered}
(X \wedge Y) Z=g(Y, Z) X-g(X, Z) Y \\
\left(X \wedge_{s} Y\right) Z=S(Y, Z) X-S(X, Z) Y \text { and } \\
(R(X, \xi) \cdot R)(U, Z) W=R(X, \xi) R(U, Z) W-R(R(X, \xi) U, Z) W \\
-R(U, R(X, \xi) Z) W-R(U, Z) R(X, \xi) W
\end{gathered}
$$

where $R(X, Y)$ is the Riemannian curvature tensor.

If we consider $(R(X, \xi) \cdot R)(U, Z) W=0$ and by putting $U=\xi$ in (2.3), we have

$$
\begin{aligned}
R(X, \xi) R(\xi, Z) W-R(R(X, \xi) \xi, Z) W-R(\xi, R(X, \xi) Z) W & -R(\xi, Z) R(X, \xi) W=0 .
\end{aligned}
$$

Now, by using (1.15) and (1.18), equation (2.4) becomes

$$
R(X, Z, W)=g(X, W) Z-g(Z, W) X .
$$

By a suitable contraction of $(2.5)$, we get $r=-n(n-1)$, where $r$ is the scalar curvature of $M_{n}$. Thus we state the following theorem.

Theorem 2.1. A P-Kenmotsu manifold with the condition $R(\xi, X) \cdot R=0$ is of constant curvature.

\section{P-Kenmotsu Manifold with the Condition $R(\xi, X) \cdot S=0$}

Let us suppose that $(R(\xi, X) . S)(Y, Z)=0$, where $S(X, Y)$ is the Ricci tensor. Then,

$$
S(R(\xi, X) Y, Z)+S(Y, R(\xi, X) Z)=0 .
$$

By putting $Z=\xi$ in (3.1) and on using the equations (1.15) and (1.18), we get

$$
S[\eta(Y) X-g(X, Y) \xi, \xi]+S[Y, X-\eta(X) \xi]=0
$$

Again, on using (1.12), we get

$$
S(X, Y)=-(n-1) g(X, Y) \quad(\text { or })
$$




$$
S(X, Y)=\frac{r}{n} g(X, Y) \text {, as the scalar curvature is } r=-n(n-1) .
$$

This proves that the manifold is an Einstein one and hence we state the following theorem.

Theorem 3.1. A P-Kenmotsu manifold with the condition $R(\xi, X) . S=0$ is an Einstein manifold.

Corollary 3.1. If a P-Kenmotsu manifold is an Einstein manifold then its scalar curvature $r$ is constant and is equal to $-n(n-1)$, and hence it is an SP-Kenmotsu manifold.

\section{Weyl-Projective Curvature Tensor on Ricci-Parallel P-Kenmotsu Manifold}

In this section, first we consider a Ricci-parallel P-Kenmotsu manifold with the condition $R(X, \xi) \cdot P=P(X, \xi) \cdot R$, where the Weyl-projective curvature tensor $P(X, Y)$ of a Riemannian manifold, of type $(1,3)$, is defined as $[8]$

$$
P(X, Y) Z=R(X, Y) Z-\frac{1}{n-1}[S(Y, Z) X-S(X, Z) Y]
$$

Definition 4.1. A P-Kenmotsu manifold is called Ricci-parallel if $\left(\nabla_{X} S\right)=$ 0 , where $S(X, Y)$ is the Ricci tensor and $\nabla$ is the covariant derivative.

The above condition implies that $S(X, Y)=-(n-1) g(X, Y)$.

Therefore, the equation (4.1) reduces to

$$
P(X, Y) Z=R(X, Y) Z+[g(Y, Z) X-g(X, Z) Y]
$$

By putting $X=\xi, Z=\xi$ in (4.2), and on using the equations (1.3) and (1.15), we get

$$
P(\xi, Y) \xi=P(Y, \xi) \xi=0, \text { for any vector field } Y \text {. }
$$

The homeomorphisms $R(X, \xi) \cdot P$ and $P(X, \xi) \cdot R$ are respectively defined as:

$$
\begin{aligned}
(R(X, \xi) \cdot P)(U, Z) W= & R(X, \xi) P(U, Z) W-P(R(X, \xi) U, Z) W \\
& -P(U, R(X, \xi) Z) W-P(U, Z) R(X, \xi) W
\end{aligned}
$$

and

$$
\begin{aligned}
(P(X, \xi) \cdot R)(U, Z) W= & P(X, \xi) R(U, Z) W-R(P(X, \xi) U, Z) W \\
& -R(U, P(X, \xi) Z) W-R(U, Z) P(X, \xi) W
\end{aligned}
$$


By putting $U=W=\xi$ in (4.4), and on using (4.3), we get

$$
(R(X, \xi) \cdot P)(\xi, Z) \xi=-P(R(X, \xi) \xi, Z) \xi-P(\xi, Z) R(X, \xi) \xi .
$$

By using the equations (4.3) and (1.15), we get

$$
P(R(X, \xi) \xi, Z) \xi=-P(X, Z) \xi \text { and } P(\xi, Z) R(X, \xi) \xi=-P(\xi, Z) X,
$$

which on substituting in (4.6) gives

$$
(R(X, \xi) \cdot P)(\xi, Z) \xi=P(X, Z) \xi+P(\xi, Z) X .
$$

Similarly, by putting $U=W=\xi$ in (4.5), we get

$$
\begin{aligned}
(P(X, \xi) \cdot R)(\xi, Z) \xi= & P(X, \xi) R(\xi, Z) \xi-R(P(X, \xi) \xi, Z) \xi \\
& -R(\xi, P(X, \xi) Z) \xi-R(\xi, Z) P(X, \xi) \xi .
\end{aligned}
$$

Also, from the equations (1.13), (1.15) and (4.3), we get

$$
\begin{aligned}
P(X, \xi) R(\xi, Z) \xi= & P(X, \xi) Z ; \quad R(P(X, \xi) \xi, Z) \xi=0 ; \\
& R(\xi, P(X, \xi) Z) \xi=P(X, \xi) Z \text { and } R(\xi, Z) P(X, \xi) \xi=0 .
\end{aligned}
$$

Therefore, from equations (4.9) and (4.10), we get

$$
(P(X, \xi) \cdot R)(\xi, Z) \xi=0 .
$$

Since $R(X, \xi) \cdot P=P(X, \xi) \cdot R$, we also have

$$
(R(X, \xi) \cdot P)(\xi, Z) \xi=0
$$

and hence from equations (4.8) and (4.12), we get

$$
P(X, Z) \xi+P(\xi, Z) X=0 .
$$

Using the equations (4.2), (1.3) and (1.17), we also have

$$
P(X, Z) \xi=0 \text { and } P(\xi, Z) X=R(\xi, Z) X+g(Z, X) \xi-\eta(X) Z \text {. }
$$

Therefore, from (4.13) and (4.14), we get $R(\xi, Z) X=\eta(X) Z-g(Z, X) \xi$ and it implies that the manifold is of constant curvature -1 . Thus we state the following theorem.

Theorem 4.1. A Ricci-parallel P-Kenmotsu manifold with the condition $R(X, \xi) \cdot P=P(X, \xi) \cdot R$ is of constant curvature -1 and consequently it is locally isomorphic to the hyperbolic space. 
Further, in this section, we consider the Ricci-parallel P-Kenmotsu manifold with the condition $R(X, \xi) \cdot P=L[(X \wedge \xi) \cdot P], L \neq-1$, where $\mathrm{L}$ is some function on $M_{n}$. That is

$$
(R(X, \xi) \cdot P)(\xi, Z) \xi=L[((X \wedge \xi) \cdot P)(\xi, Z) \xi]
$$

where

$$
\begin{aligned}
L[((X \wedge \xi) . P)(\xi, Z) \xi]= & L[(X \wedge \xi) P(\xi, Z) \xi-P((X \wedge \xi) \xi, Z) \xi \\
& -P(\xi,(X \wedge \xi) Z) \xi-P(\xi, Z)(X \wedge \xi) \xi] .
\end{aligned}
$$

Using (4.3) and (2.1), we get

$$
\begin{aligned}
(X \wedge \xi) P(\xi, Z) \xi=0 ; P((X \wedge \xi) \xi, Z) \xi & =P(X, Z) \xi \\
P(\xi,(X \wedge \xi) Z) \xi=0 \text { and } P(\xi, Z)(X \wedge \xi) \xi & =P(\xi, Z) X
\end{aligned}
$$

and hence the equation (4.16) becomes

$$
L[((X \wedge \xi) . P)(\xi, Z) \xi]=L[P(X, Z) \xi+P(\xi, Z) X] .
$$

By using (4.8) and (4.18), equation (4.15) reduces to

$$
(1+L)[P(X, Z) \xi+P(\xi, Z) X]=0 .
$$

Then on using equations (4.2),(1.3) and (1.17), we get from (4.19) that

$$
(1+L)[R(\xi, Z) X-\eta(X) Z+g(X, Z) \xi]=0 .
$$

Since $L \neq-1$, we have $R(\xi, Z) X=\eta(X) Z-g(X, Z) \xi$ and it implies that the manifold $M_{n}$ is of constant curvature -1 . Thus we state the following result.

Theorem 4.2. A Ricci-parallel P-Kenmotsu manifold with the condition

$$
R(X, \xi) \cdot P=L[(X \wedge \xi) \cdot P] \quad(L \neq-1)
$$

is of constant curvature -1 and consequently it is locally isomorphic to the hyperbolic space.

\section{Conclusion}

In this paper, we obtain certain curvature properties of Ricci-parallel paraKenmotsu manifolds with the conditions

$$
R(X, \xi) \cdot P=P(X, \xi) \cdot R \text { and } R(X, \xi) \cdot P=L[(X \wedge \xi) \cdot P],
$$

$L \neq-1$. Some of the results obtained in this paper are in similar to the results reported earlier in the case of para-Sasakian manifolds $[9,10]$. 


\section{Acknowledgments}

The authors acknowledge Prof. Kalpana of Banaras Hindu University, Dr. B. Satyanarayana of Nagarjuna University and Mr. T. Satyanarayana of Pragathi Engineering College for their valuable suggestions in preparation of the manuscript.

\section{References}

[1] I. Sato, On a structure similar to the almost contact structure, Tensor (N.S.), 30 (1976), 219-224.

[2] T. Adati and K. Matsumoto, On conformally recurrent and conformally symmetric PSasakian manifolds, TRU Math., 13 (1977), 25-32.

[3] K. Kenmotsu, A class of almost contact Riemannian manifolds, Tohoku Math. Journal, 24 (1972), 93-103, doi: 10.2748/tmj/1178241594.

[4] B. B. Sinha and K. L. Sai Prasad, A class of almost para contact metric Manifold, Bulletin of the Calcutta Mathematical Society, 87 (1995), 307-312.

[5] K. L. Sai Prasad and T. Satyanarayana, On para-Kenmotsu manifolds satisfying certain conditions on the curvature tensors, Advances in Applied Science Research, 6, No. 4 (2015), 108-113.

[6] T. Satyanarayana and K. L. Sai Prasad, On semi-symmetric para Kenmotsu manifolds, Turkish J. of Analysis and Number Theory, 3, No. 5 (2015), 145-148, doi: 10.12691/tjant3-6-1.

[7] I. Sato and K. Matsumoto, On P-Sasakian satisfying certain conditions, Tensor (N.S.), 33 (1979), 173-178.

[8] G. P. Pokhariyal and R. S. Mishara, The curvature tensors and their relativistic significance, Yokohoma Math. J., 18 (1970), 105-108.

[9] Dhruwa Narain and G. N. Tripathi, On Para-Sasakian manifolds, Journal of International Academy of Phy. Sciences., 13, No. 3 (2009), 261-267.

[10] G. N. Tripathi and Dhruwa Narain, Concircular and projective curvature tensors on P-Sasakian manifold, Journal of Rajasthan Academy of Phy. Sci., 13, No. 3-4 (2014), 213-220. 